\title{
Genetic Variants, Circulating Level of MCPI with Risk of Chronic Obstructive Pulmonary Disease: A Case-Control Study
}

\author{
Chunyi Lin ${ }^{1, *}$ \\ Zhimin Wang ${ }^{2, *}$ \\ Lu Shen' \\ Gao Yil \\ Meichan $\mathrm{Li}^{\prime}$ \\ Defu Li ${ }^{1}$
}

'Respiratory Medicine, The Fifth Affiliated Hospital of Guangzhou Medical University, Guangzhou, 5I0700, People's Republic of China; ${ }^{2}$ Intensive Care Unit (ICU), The Fifth Affiliated Hospital of Guangzhou Medical University,

Guangzhou, 510700, People's Republic of China

*These authors contributed equally to this work
Correspondence: Lu Shen

Respiratory Medicine, The Fifth Affiliated Hospital of Guangzhou Medical

University, Guangzhou, 510700, People's

Republic of China

$\mathrm{Tel} / \mathrm{Fax}+86-20-85959110$

Email ccc0987I@2Icn.com
Background: Chronic obstructive pulmonary disease (COPD) ranks one of the major causes of mortality worldwide. Inflammation is greatly involved in the pathogenesis of COPD. Monocyte chemoattractant protein-1 (MCP1) has been implicated to play an important role in the inflammatory response of various pathological processes.

Methods: In this study, we conducted a hospital-based case-control study in a Chinese population, aiming to evaluate the potential associations of genetic polymorphisms of the MCP1 gene (rs1024611, rs2857656, and rs4586) and circulating level of MCP1 with COPD risk.

Results: We found that rs1024611 (OR=1.37;95\% CI=1.11-1.69; $P$-value=0.004) and rs4586 (OR=1.33; 95\% CI=1.09-1.63; $P$-value=0.006) were significantly associated with increased COPD risk. In the dominant model, both $\mathrm{rs} 1024611$ (OR=1.46; 95\% CI=1.11-1.92; $P$-value $=0.006)$ and $\mathrm{rs} 4586(\mathrm{OR}=1.56 ; 95 \% \mathrm{CI}=1.18-2.07 ; P$-value $=0.002)$ were significantly associated with increased COPD risk. Genotypes of rs1024611 and rs4586 with minor alleles had a significantly higher circulating level of MCP1 $(P<0.001)$. Meanwhile, a circulating level of MCP1 was significantly associated with increased COPD risk (OR for per quartile increment $=1.35,95 \% \mathrm{CI}=1.21-1.52, P<0.001$ ).

Conclusion: Our study indicated that genetic polymorphisms of the MCP1 gene and circulating level of MCP1 contributed to the COPD risk in the Chinese population. MCP1 contributed importantly to the pathophysiological process and occurrence of COPD.

Keywords: COPD, variation, genetic, MCP1, case-control study

\section{Introduction}

Chronic obstructive pulmonary disease (COPD) is one of the major causes of mortality worldwide, which is characterized by troubles of persistent tract symptoms, incomplete reversible airflow obstruction, chronic inflammation, and a progressive declined lung function. ${ }^{1,2}$ COPD, resulting in 3197.8 thousand allage deaths globally, ranks the first cause of mortality in chronic respiratory diseases. ${ }^{3}$ Many endogenous factors including inflammatory processes and genetic predisposition, and exogenous factors including smoking and air pollution, were involved in the pathogenesis of COPD. ${ }^{4}$

Chemokines, small heparin-binding proteins that direct the movement of circulating leukocytes towards sites of inflammation, were tightly involved in all stages of COPD evolution, including chronic inflammation and bronchial remodeling. ${ }^{5-7}$ One of the best characterized chemokines, monocyte chemoattractant protein-1 
(MCP1; also known as chemokine ligand (C-C motif) 2; CCL2), has been implicated to play an important role in the inflammatory response during various pathological processes. $^{8-13}$ The circulating level of MCP1 has been linked to cardiovascular mortality, stroke, cancers, and atrial fibrillation. ${ }^{14-18}$ Genetic polymorphisms of the MCP1 gene have also been explored for their associations with various inflammation related diseases and phenotypes, including chronic periodontitis in end-stage renal disease, obstructive sleep apnea syndrome, diabetic retinopathy in type 2 diabetes mellitus, rheumatoid arthritis, tuberculosis, lupus nephritis, coronary artery disease, and COPD. ${ }^{19-26}$ The most studies of SNPs included SNP rs1024611 (-2518 A>G), rs2857656 (-362 G>C), and rs4586 (Cys35Cys). The associations of genetic polymorphisms of the MCP1 gene with COPD risk were not systematically explored to date. Only two studies evaluated the association between SNP rs1024611 and risk of CPOD, however, the results were inconsistent. ${ }^{25,26}$

Considering the important role of the chronic inflammation and inflammatory chemotaxis of MCP1 in the pathophysiological process of COPD, here we conducted a hospital-based case-control study in a Chinese population, which aimed to evaluate the potential associations of genetic polymorphisms of the MCP1 gene (rs1024611, rs2857656, and rs4586) and circulating level of MCP1 with COPD risk.

\section{Patients and Methods}

\section{Study Subjects}

Totally included in this study were 450 acute COPD cases and 500 frequency-matched healthy controls by age, gender, ethnicity, and living area. COPD was diagnosed using pulmonary function tests according to criteria of the Global Initiative for Chronic Obstructive Lung Disease (GOLD) that post-bronchodilator forced expiratory volume during the first second (FEV1)/forced vital capacity (FVC) values $<0.7$ for the first time. Cases with lung cancer, asthma, tuberculosis, bronchiectasis, pulmonary fibrosis, pulmonary cystic fibrosis, etc. were excluded. All participants accepted a physical examination, a chest $\mathrm{X}$-ray, and a pulmonary function test. Demographic and clinical information were collected from all individuals using a uniformed questionnaire, and $5 \mathrm{msL}$ of peripheral blood was drawn from each enrolled person. All subjects provided written informed consent before they participate in this research, the study protocol was approved by the ethical committee of the Fifth Affiliated Hospital of Guangzhou Medical University (\#00012), and the study was conducted in accordance with the Declaration of Helsinki.

\section{Genotyping and Biochemical Analyses}

Blood samples were stored at $-80^{\circ} \mathrm{C}$ before analyses. Genomic DNA was extracted from whole blood samples using the commercial QIAamp Genomic DNA isolation kit. The DNA was then quantified using a NanoDrop system (Thermo Fisher Scientific, Wilmington, DE, USA). Genotyping of three MCP1 polymorphisms (rs1024611, rs2857656, and rs4586) was conducted using the Agena MassARRAY system (Agena, San Diego, CA, USA). We performed the data sorting and analyses by Agena Bioscience TYPER 4.0 software. For quality control, 2\% of the samples were genotyped in duplicate as controls for allele assignment. Serum MCP-1 levels were determined by a multiplex assay using the Bioplex Suspension Array (Bio-Rad) according to the manufacturer's specifications (The limit of detection for all target cytokines was $0.2-$ $32,000 \mathrm{pg} / \mathrm{mL}$ ). The laboratory personnel was blind to the disease status of the participants.

\section{Statistical Analysis}

All the statistical analyses were evaluated using SAS 9.4 software (SAS Institute, Cary, NC, USA). $P<0.05$ was considered statistically significant. Continuous variables are expressed as mean \pm standard deviation or median with interquartile range, and compared by the Student's $t$-test or Mann-Whitney $U$-test. Categorical data are presented as frequency and percentage, and analyzed using chi-square test. Odds ratio (OR) with 95\% confidence interval $(95 \% \mathrm{CI})$ for the associations of COPD risk with MCP1 gene polymorphisms and quartiles of MCP1 were calculated using a binary logistic regression model. Chi square test was used to estimate the goodness of fit of Hardy Weinberg equilibrium expectation of control samples. Quartiles of MCP1 distribution among controls were used to convert it to a categorical variable.

\section{Results}

\section{Participant Characteristics}

Table 1 presents the demographic characteristics of study participants, including 450 acute COPD cases and 500 frequency-matched (by age and gender) healthy controls. The distributions of the age $(P=0.923)$, gender $(P=0.916)$, 
Table I Demographics of Study Participants

\begin{tabular}{|l|c|c|c|}
\hline & $\begin{array}{c}\text { COPD Cases } \\
(\mathbf{N}=\mathbf{4 5 0})\end{array}$ & $\begin{array}{c}\text { Controls } \\
\mathbf{( N = 5 0 0 )}\end{array}$ & P-value \\
\hline $\begin{array}{l}\text { Age (years) } \\
\leq 60\end{array}$ & $139(30.9 \%)$ & $153(30.6 \%)$ & 0.923 \\
$>60$ & $311(69.1 \%)$ & $347(69.4 \%)$ & \\
\hline Gender & & & \\
Male & $320(71.1 \%)$ & $354(70.8 \%)$ & 0.916 \\
Female & $130(28.9 \%)$ & $146(29.2 \%)$ & \\
BMI (kg/m $\left.{ }^{2}\right)$ & $23.3 \pm 3.11$ & $23.6 \pm 3.14$ & 0.140 \\
\hline Smoking status & & $156(31.2 \%)$ & $<0.00 \mathrm{I}$ \\
Ever & $270(60.0 \%)$ & $344(68.8 \%)$ & $<$ \\
Never & $180(40.0 \%)$ & $157.1(126.8-186.7)$ & $<0.00 \mathrm{I}$ \\
MCP-I, pg/mL & $175.6(141.6-21 \mathrm{I} .6)$ & $0.81 \pm 0.10$ & $<0.00 \mathrm{I}$ \\
FEVI/FVC & $0.55 \pm 0.09$ & & \\
\hline Gold grades & & & \\
A and B & $237(52.7 \%)$ & & \\
C and D & $213(47.3 \%)$ & & \\
\hline
\end{tabular}

Note: Bold values are statistically significant $(P<0.05)$.

Abbreviation: BMl, body mass index.

and body mass index (BMI, $P=0.140$ ) were not statistically significant, which indicated the comparability between the case and control groups. However, we found that the COPD cases were more likely to be ever smokers, and had a significantly lower FEV1/FVC value $(P<0.001)$. Of the cases, $52.7 \%$ were graded $\mathrm{A}$ or $\mathrm{B}$ according to the GOLD criteria. The circulating level of MCP1 in the COPD cases was significantly higher than that in the control group (median=175.6 vs $157.1, P<0.001)$.

\section{Associations of MCPI Gene Polymorphisms with COPD Risk and Circulating Level of MCPI}

As shown in Table 2, the genotype frequencies and associations of three MCP1 polymorphisms (rs1024611, rs2857656, and rs4586) with COPD risk were presented and evaluated. The genotype distribution of all three polymorphisms in the controls were in line with HardyWeinberg equilibrium $(P>0.05)$. Logistic regression analysis showed rs1024611 (OR=1.37; 95\% CI=1.11-1.69; $P=0.004)$ and rs4586 (OR=1.33; 95\% $\mathrm{CI}=1.09-1.63$; $P=0.006)$ were significantly associated with increased COPD risk. In the dominant model, both rs1024611 $(\mathrm{OR}=1.46 ; 95 \% \mathrm{CI}=1.11-1.92 ; P=0.006)$ and $\mathrm{rs} 4586$ $(\mathrm{OR}=1.56 ; 95 \% \mathrm{CI}=1.18-2.07 ; P=0.002)$ were significantly associated with increased COPD risk. However,
Table 2 Logistic Regression Analysis of Associations between MCPI Gene Polymorphisms and Risk of COPD

\begin{tabular}{|c|c|c|c|c|}
\hline Variants & $\begin{array}{c}\text { Cases } \\
(n=450)\end{array}$ & $\begin{array}{l}\text { Controls } \\
(n=500)\end{array}$ & OR $(95 \% \mathrm{Cl})^{*}$ & $P$ \\
\hline rs $102461 \mathrm{I}$ & & & & \\
\hline AA & 195 & 259 & 1.00 (reference) & \\
\hline AG & 204 & 201 & $1.40(1.05-1.87)$ & 0.022 \\
\hline GG & 51 & 40 & $1.76(1.11-2.79)$ & 0.016 \\
\hline$G$ vs $A$ & & & $1.37(1.11-1.69)$ & 0.004 \\
\hline Dominant model & & & $1.46(1.11-1.92)$ & 0.006 \\
\hline Recessive model & & & $1.53(0.97-2.4 I)$ & 0.068 \\
\hline rs 2857656 & & & & \\
\hline GG & 216 & 241 & 1.00 (reference) & \\
\hline CG & 207 & 221 & $1.09(0.68-1.75)$ & 0.731 \\
\hline $\mathrm{CC}$ & 27 & 38 & $0.82(0.54-1.25)$ & 0.364 \\
\hline$C$ vs $G$ & & & $1.01(0.98-1.04)$ & 0.726 \\
\hline Dominant model & & & $1.05(0.25-4.39)$ & 0.948 \\
\hline Recessive model & & & $0.81(0.54-1.22)$ & 0.306 \\
\hline rs4586 & & & & \\
\hline $\mathrm{CC}$ & 132 & 192 & 1.00 (reference) & \\
\hline CT & 235 & 230 & $1.55(1.15-2.09)$ & 0.004 \\
\hline TT & 83 & 78 & $1.61(1.09-2.39)$ & 0.018 \\
\hline T vs $C$ & & & $1.33(1.09-1.63)$ & 0.006 \\
\hline Dominant model & & & $1.56(1.18-2.07)$ & 0.002 \\
\hline Recessive model & & & $1.27(0.87-1.87)$ & 0.221 \\
\hline
\end{tabular}

Notes: *Adjusted for age, gender, BMl, and smoking status; bold values are statistically significant $(P<0.05)$.

no statistically significant association was detected for SNP rs2857656. Stratified analyses by Gold grades showed rs1024611 and rs4586 was still associated with increased COPD risk in dominant model in patients with grade A or B, while rs4586 was associated with increased COPD risk in the dominant model in patients with grade $\mathrm{C}$ or D (Table 3). Figure 1 presented the associations between MCP-1 SNPs and circulating level of MCP1 in healthy controls. Genotypes of rs1024611 and rs4586 with minor alleles had a significantly higher level of serum MCP-1 $(P<0.001)$.

\section{Associations between Quartiles of MCPI and COPD Risk}

We also analyzed the associations between quartiles of MCP1 (quartiles of MCP1 distribution among controls were used to convert it to a categorical variable) and COPD risk using the binary logistic regression model adjusted for age, gender, BMI, and smoking status (Table 4). The results showed that circulating level of MCP1 was significantly associated with increased COPD risk $(\mathrm{OR}$ for per quartile increment $=1.35,95 \%$ 
Table 3 Logistic Regression Analysis of Associations between MCPI Gene Polymorphisms and Risk of COPD, Stratified by Gold Grades

\begin{tabular}{|c|c|c|c|c|}
\hline \multirow[b]{2}{*}{ Variants } & \multicolumn{2}{|c|}{ GOLD A and B } & \multicolumn{2}{|c|}{ GOLD $C$ and $D$} \\
\hline & OR $(95 \% \mathrm{Cl}) *$ & $P$ & OR $(95 \% \mathrm{Cl}) *$ & $P$ \\
\hline \multicolumn{5}{|l|}{ rsI0246II } \\
\hline $\mathrm{AA}$ & 1.00 (reference) & & 1.00 (reference) & \\
\hline AG & $1.38(0.99-1.91)$ & 0.055 & I.32 (0.94-I.84) & 0.111 \\
\hline GG & $1.94(1.15-3.27)$ & 0.012 & $1.43(0.8-2.55)$ & 0.222 \\
\hline$G$ vs $A$ & $1.39(1.1-1.76)$ & 0.005 & I.24 (0.97-I.58) & 0.086 \\
\hline Dominant model & $1.53(1.1-2.12)$ & 0.011 & $1.39(0.98-1.97)$ & 0.065 \\
\hline Recessive model & $1.73(1.04-2.89)$ & 0.035 & I.3I (0.7I-2.43) & 0.394 \\
\hline \multicolumn{5}{|l|}{ rs2857656 } \\
\hline GG & I.00 (reference) & & 1.00 (reference) & \\
\hline CG & $\mathrm{I} .02(0.74-\mathrm{I} .4 \mathrm{I})$ & 0.881 & $1.07(0.77-1.49)$ & 0.696 \\
\hline $\mathrm{CC}$ & $0.66(0.33-1.3)$ & 0.226 & $0.95(0.5-1.8 I)$ & 0.879 \\
\hline C vs $G$ & $0.92(0.72-1.18)$ & 0.517 & $1.02(0.79-1.3)$ & 0.896 \\
\hline Dominant model & $1.01(0.92-1.11)$ & 0.843 & $1.09(0.63-1.89)$ & 0.748 \\
\hline Recessive model & $0.67(0.38-1.2)$ & 0.180 & $0.96(0.7-1.31)$ & 0.785 \\
\hline \multicolumn{5}{|l|}{ rs4586 } \\
\hline $\mathrm{CC}$ & I.00 (reference) & & 1.00 (reference) & \\
\hline CT & $1.54(1.09-2.2)$ & 0.016 & $1.43(0.99-2.04)$ & 0.054 \\
\hline TT & $1.69(1.07-2.67)$ & 0.024 & $1.4(0.87-2.27)$ & 0.169 \\
\hline T vs C & $1.33(1.07-1.66)$ & 0.011 & $1.22(0.97-1.54)$ & 0.089 \\
\hline Dominant model & $1.64(1.16-2.32)$ & 0.005 & $1.48(1.03-2.12)$ & 0.035 \\
\hline Recessive model & $1.36(0.87-2.1)$ & 0.176 & $1.18(0.69-2.02)$ & 0.537 \\
\hline
\end{tabular}

Notes: *Adjusted for age, gender, BMI, and smoking status; bold values are statistically significant $(P<0.05)$.

$\mathrm{CI}=1.21-1.52, P<0.001)$. Compared with subjects in the first quartile of MCP1 level, those in the second quartile $(\mathrm{OR}=1.40 ; 95 \% \quad \mathrm{CI}=0.94-2.08)$, the third quartile $(\mathrm{OR}=1.40 ; 95 \% \mathrm{CI}=0.94-2.08)$, and the fourth quartile $(\mathrm{OR}=2.63 ; 95 \% \mathrm{CI}=1.82-3.81)$ were all associated with increased risk of COPD.

\section{Discussion}

The current study was conducted to evaluate whether genetic polymorphisms of the MCP1 gene (rs1024611, rs2857656, and rs4586) and circulating level of MCP1 contributed to the COPD risk in a Chinese population. We identified that SNP rs1024611 and rs4586 were significantly associated with increased COPD risk. Genotypes of rs1024611 and rs4586 with minor alleles had significantly higher levels of serum MCP-1. Further, we found the circulating level of MCP1 was associated with 1.35fold increased COPD risk. Taking the findings above together, MCP1 contributes importantly to the pathophysiological process and occurrence of COPD. To our knowledge, this should be the most comprehensive study exploring serological and genetic associations of MCP1 with COPD risk.

Genetic polymorphisms of the MCP1 gene have been explored in many diseases and phenotypes. Among them, MCP1 rs1024611 was the most studied SNP of the MCP1 gene, although the findings were inconsistent. Kerget et al20 found that OSAS patients with homozygous MCP1 rs1024611 SNP are at higher risk for CAD. SNP rs1024611 and rs4586 conferred the susceptibility to osteoarthritis and may be potential markers for early diagnosis of osteoarthritis. ${ }^{27}$ However, a meta-analysis by Wang et $\mathrm{al}^{28}$ reported that MCP1 rs1024611 might not be associated with genetic susceptibility of $\mathrm{AD}$ in the general population. SNP rs1024611 was previously evaluated for its association with COPD in Taiwanese men, with 67 COPD cases and 58 controls, but they only got null results in all genetic models. ${ }^{25}$ However, rs1024611 was associated with a 1.37 -fold increased risk of COPD in our study. The difference may be caused by the small sample 

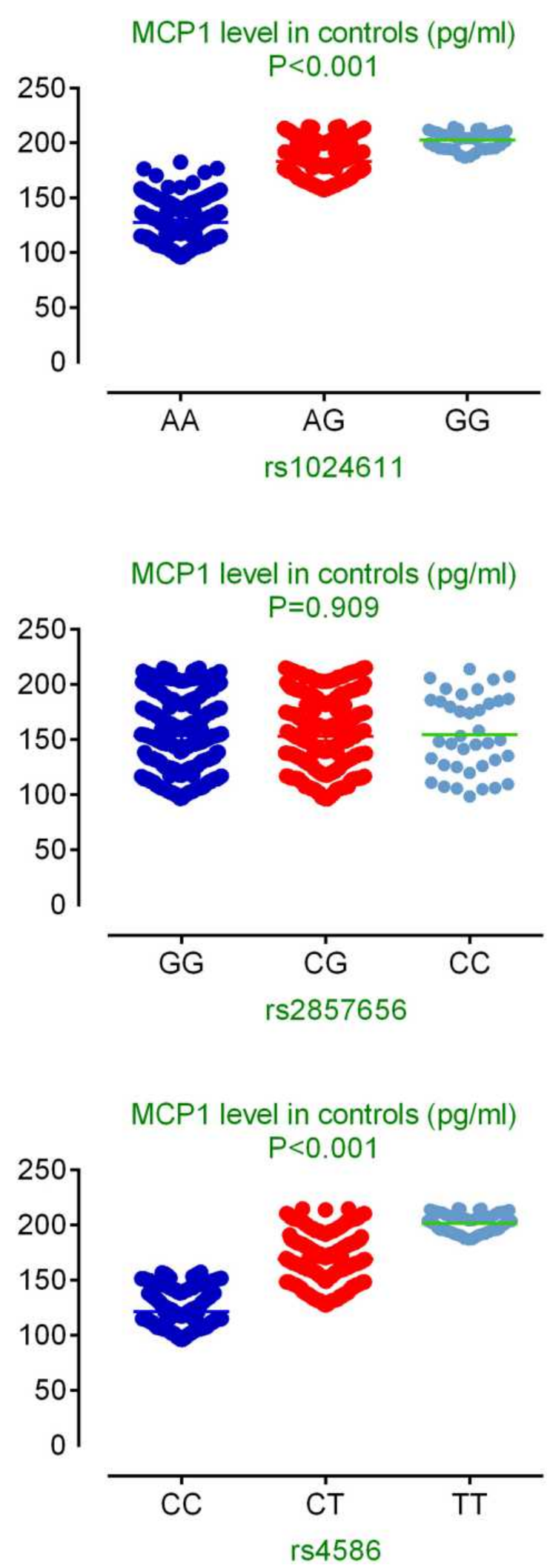

Figure I Associations between MCP-I SNPs and circulating level of MCPI in healthy controls. size and limited statistical power. Calculated by Quanto software (version 1.2.4), we have $88.7 \%$ statistical power to detect such an association between SNP rs1024611 and COPD risk.

MCP1 rs4586, which results in a synonymous variant, was another most studied SNP of the MCP1 gene. Xu et $\mathrm{al}^{27}$ reported SNP rs4586 was significantly associated with the risk of osteoarthritis, and could serve as a potential marker for early diagnosis of osteoarthritis. However, $\mathrm{Ma}$ et $\mathrm{al}^{29}$ found that $\mathrm{rs} 4586 \mathrm{~T}$ was a protective factor for risk of chronic postsurgical pain. In our study, we identified that rs4586 was associated with a 1.33-fold increased risk of COPD, and the statistical power was $86.4 \%$ by Quanto software (version 1.2.4). Using GTEX portal, we found rs1024611 and rs4586 functioned as expression quantitative trait loci (eQTL), and were significantly associated with increased mRNA levels of CCL13 in different human tissues, another important chemokine in the biological course of inflammatory chemotaxis. ${ }^{30}$

Some limitations should be acknowledged when interpreting the results. First, the current study included several phenotypes of COPD, such as smoking-COPD, nonsmoking COPD, etc., although the smoking status was used as the adjusting variable. Further studies should focus on more accurate classification for a specific type of COPD. Second, our samples comprised exclusively Chinese Han population, thus the findings cannot be generalized to other ethnic groups. Third, we only genotyped three functional variants in the MCP1 gene, which might under-evaluate the contribution of this gene to the pathogenesis of COPD. Further fine mapping studies are warranted for exploration of the exact causal SNPs. Fourth, the controls were recruited from the outpatient department of the hospital, which may lead to selection bias, although the frequency-match strategy in the case-control study design has ensured the comparability between the case and control groups.

Taken together, our study indicates that genetic polymorphisms of the MCP1 gene and circulating level of MCP1 contributed to the COPD risk in a Chinese population. Validation with a larger sample size from different ethnic groups are warranted, and further investigations are required to elucidate immunopathogenic mechanisms of MCP1 in the occurrence of COPD. 
Table 4 Distribution of Quartiles of MCPI in COPD Cases and Controls

\begin{tabular}{|c|c|c|c|c|}
\hline IFN- $\gamma(\mathrm{pg} / \mathrm{mL})$ & Cases & Controls & OR (95\% Cls)* & $P$-value \\
\hline Quartile I $(\leq 126.8)$ & 70 & 125 & reference & \\
\hline Quartile 2 (|26.8-157.I) & 98 & 125 & $1.40(0.94-2.08)$ & 0.094 \\
\hline Quartile 3 (157.I-186.7) & 98 & 125 & $1.40(0.94-2.08)$ & 0.094 \\
\hline Quartile 4 (> |86.7) & 184 & 125 & $2.63(\mathrm{I} .82-3.8 \mathrm{I})$ & $<0.001$ \\
\hline Per quartile & & & $1.35(1.21-1.52)$ & $<0.001$ \\
\hline
\end{tabular}

Notes: *Adjusted for age, gender, BMI, and smoking status; bold values are statistically significant $(P<0.05)$.

\section{Disclosure}

The authors declare that they have no conflicts of interest.

\section{References}

1. Ruvuna L, Sood A. Epidemiology of chronic obstructive pulmonary disease. Clin Chest Med. 2020;41:315-327. doi:10.1016/j. ccm.2020.05.002

2. Diaz-Guzman E, Mannino DM. Epidemiology and prevalence of chronic obstructive pulmonary disease. Clin Chest Med. 2014;35:7-16. doi:10.1016/j.ccm.2013.10.002

3. Roth GA, Abate D, Abate KH, et al. Global, regional, and national age-sex-specific mortality for 282 causes of death in 195 countries and territories, 1980-2017: a systematic analysis for the Global Burden of Disease Study 2017. Lancet. 2018;392:1736-1788.

4. Yu T, Niu W, Niu H, et al. Chitinase 3-like 1 polymorphisms and risk of chronic obstructive pulmonary disease and asthma in a Chinese population. J Gene Med. 2020;22:e3208. doi:10.1002/jgm.3208

5. Henrot P, Prevel R, Berger P, et al. Chemokines in COPD: from implication to therapeutic use. Int $J$ Mol Sci. 2019;20:2785. doi:10.3390/ijms20112785

6. Nucera F, Lo Bello F, Shen SS, et al. Role of atypical chemokines and chemokine receptors pathways in the pathogenesis of COPD. Curr Med Chem. 2020. doi:10.2174/0929867327999200819145327

7. Lim SB, Di Lee W, Vasudevan J, et al. Liquid biopsy: one cell at a time. NPJ Precis Oncol. 2019;3:23. doi:10.1038/s41698-019-0095-0

8. de Boer WI, Sont JK, van Schadewijk A, et al. Monocyte chemoattractant protein 1 , interleukin 8 , and chronic airways inflammation in COPD. J Pathol. 2000;190:619-626. doi:10.1002/(SICI)1096-9896(200004)190:5<619::AID-PATH555>3.0.CO;2-6

9. Chow FY, Nikolic-Paterson DJ, Ma FY, et al. Monocyte chemoattractant protein-1-induced tissue inflammation is critical for the development of renal injury but not type 2 diabetes in obese $\mathrm{db} / \mathrm{db}$ mice. Diabetologia. 2007;50:471-480. doi:10.1007/s00125-0060497-8

10. Labbe K, Danialou G, Gvozdic D, et al. Inhibition of monocyte chemoattractant protein-1 prevents diaphragmatic inflammation and maintains contractile function during endotoxemia. Crit Care. 2010;14:R187. doi:10.1186/cc9295

11. Onoda M, Yoshimura K, Aoki H, et al. Lysyl oxidase resolves inflammation by reducing monocyte chemoattractant protein-1 in abdominal aortic aneurysm. Atherosclerosis. 2010;208:366-369. doi:10.1016/j.atherosclerosis.2009.07.036

12. Chao J, Donham P, van Rooijen N, et al. Monocyte chemoattractant protein-1 released from alveolar macrophages mediates the systemic inflammation of acute alveolar hypoxia. Am J Respir Cell Mol Biol. 2011;45:53-61. doi:10.1165/rcmb.2010-0264OC

13. Lee YG, Jeong JJ, Nyenhuis S, et al. Recruited alveolar macrophages, in response to airway epithelial-derived monocyte chemoattractant protein $1 / \mathrm{CCl} 2$, regulate airway inflammation and remodeling in allergic asthma. Am J Respir Cell Mol Biol. 2015;52:772-784. doi: $10.1165 / \mathrm{rcmb} .2014-0255 \mathrm{OC}$
14. Georgakis MK, de Lemos JA, Ayers C, et al. Association of circulating monocyte chemoattractant protein-1 levels with cardiovascular mortality: a meta-analysis of population-based studies. JAMA Cardiol. 2020. doi:10.1001/jamacardio.2020.5392

15. Georgakis MK, Malik R, Bjorkbacka H, et al. Circulating monocyte chemoattractant protein-1 and risk of stroke: meta-analysis of population-based studies involving 17180 individuals. Circ Res. 2019;125:773-782. doi:10.1161/CIRCRESAHA.119.315380

16. Li S, Xu Y, Zhang Y, et al. Mendelian randomization analyses of genetically predicted circulating levels of cytokines with risk of breast cancer. NPJ Precis Oncol. 2020;4:25. doi:10.1038/s41698020-00131-6

17. Zhang G, Abuduoufu A, Zhou X, et al. Monocyte chemoattractant protein-1-induced protein in age-related atrial fibrillation and its association with circulating fibrosis biomarkers. Cardiology. 2019;142:244-249. doi:10.1159/000499932

18. Talbert EE, Lewis HL, Farren MR, et al. Circulating monocyte chemoattractant protein-1 (MCP-1) is associated with cachexia in treatment-naive pancreatic cancer patients. J Cachexia Sarcopenia Muscle. 2018;9:358-368. doi:10.1002/jcsm.12251

19. Ksiazek K, Buraczynska M. Association between monocyte chemoattractant protein-1-2518 (A/G) single nucleotide polymorphism and chronic periodontitis in end-stage renal disease patients - a case-control study. Immunol Invest. 2020;49:897-906. doi:10.1080/ 08820139.2019.1702052

20. Kerget B, Araz O, Erdem HB, et al. The frequency of monocyte chemoattractant protein-1 gene polymorphism in obstructive sleep apnea syndrome. Lung. 2019;197:585-592. doi:10.1007/s00408019-00256-x

21. Martin ES, Schneeberger EE, Aranda FM, et al. The -2518 A/G polymorphism in the monocyte chemoattractant protein 1 gene (MCP-1) is associated with an increased risk of rheumatoid arthritis in Argentine patients. Clin Rheumatol. 2016;35:3057-3061.

22. Tian G, Li X, Li H, et al. Systematic meta-analysis of the association between monocyte chemoattractant protein-1-2518A/G polymorphism and risk of tuberculosis. Genet Mol Res. 2015;14:5501-5510. doi:10.4238/2015.May.25.1

23. Mohammad LA, Atef DM, Abul-Saoud AM. Association of monocyte chemoattractant protein 1 (MCP-1) gene polymorphism with lupus nephritis in Egyptian patients. Hum Immunol. 2015;76:724-728. doi:10.1016/j.humimm.2015.09.027

24. Bai XY, Li S, Wang M, et al. Association of monocyte chemoattractant protein-1 (MCP-1)-2518A $>$ G polymorphism with susceptibility to coronary artery disease: a meta-analysis. Ann Hum Genet. 2015;79:173-187. doi:10.1111/ahg.12105

25. Liu SF, Wang CC, Fang WF, et al. MCP1-2518 polymorphism and chronic obstructive pulmonary disease in Taiwanese men. Exp Lung Res. 2010;36:277-283. doi:10.3109/01902140903575989

26. Bai J, Song H, Cai C, et al. The association of monocyte chemotactic protein-1 and $\mathrm{CC}$ chemokine receptor 2 gene variants with chronic obstructive pulmonary disease. DNA Cell Biol. 2012;31:1058-1063. doi:10.1089/dna.2011.1520 
27. Xu Z, Li J, Yang H, et al. Association of CCL2 gene variants with osteoarthritis. Arch Med Res. 2019;50:86-90. doi:10.1016/j. arcmed.2019.05.014

28. Wang $\mathrm{Y}$, Huang $\mathrm{S}$, Wu $\mathrm{X}$, et al. Correlation between MCP$1-2518 \mathrm{~A} / \mathrm{G}$ polymorphism and the risk of Alzheimer's disease. J Neural Transm. 2018;125:1781-1786. doi:10.1007/s00702-0181936-7
29. Ma G, Yang J, Zhao B, et al. Correlation between CCL2, CALCA, and CX3CL1 gene polymorphisms and chronic pain after cesarean section in Chinese Han women: a case control study. Medicine. 2019;98:e16706. doi:10.1097/MD.0000000000016706

30. Lonsdale J, Thomas J, Salvatore M, et al. The genotype-tissue expression (GTEx) project. Nat Genet. 2013;45:580-585. doi:10.1038/ng.2653

\section{Publish your work in this journal}

Pharmacogenomics and Personalized Medicine is an international, peer-reviewed, open access journal characterizing the influence of genotype on pharmacology leading to the development of personalized treatment programs and individualized drug selection for improved safety, efficacy and sustainability. This journal is indexed on the American Chemical Society's Chemical Abstracts Service (CAS). The manuscript management system is completely online and includes a very quick and fair peer-review system, which is all easy to use. Visit http://www.dovepress.com/testimonials.php to read real quotes from published authors. 